\title{
Million per Microliter
}

National Cancer Institute

\section{Source}

National Cancer Institute. Million per Microliter. NCI Thesaurus. Code C67308.

A derived unit expressed as a number of entities or quantity in millions per unit of volume equal to one microliter. 